\title{
Vision Rope AtTRibute MEASUREMENT SENSOR FOR GONDOLA-TYPED FACADE ROBOT ${ }^{1}$
}

\author{
Hwadong Sun ${ }^{1}$, Dong Yeop Kim ${ }^{1}$, Joon Ho Kwon ${ }^{1}$, Bong-Seok Kim ${ }^{1}$ \\ and Chang-Woo Park ${ }^{1}$ \\ ${ }^{1}$ Intelligent Robotics Research Center, Korea Electronics Technology Institute, Bucheon- \\ si, Gyeonggido, Republic of Korea \\ sword32@keti.re.kr
}

\begin{abstract}
The purpose of robotics and automation in construction is better working condition of human labours. Safety hazards of high-rise buildings have been cruel for decades. For example, falls of gondola at building sites have carried off a lot of human lives. As an important part of this automation, we proposed an approach to sense pose of the gondola in this paper. A vision camera measures the attributes of the wire rope, and the attributes are converted to the pose of the gondola cage. The pose of the gondola cage is $X, Y$ and $Z$ axis and rotation (Euler angles) of the gondola. Because we use only one CCD camera, the sensor system is compact and inexpensive.
\end{abstract}

\section{KEYWORDS}

gondola robot, building facade, balance, rope attribute

\section{INTRODUCTION}

The building facade maintenance using robotic system has following merits. First, human labours can be out of dangerous of falling accident. Now, workers on the building facade have used a rope to hold their bodies in the air during end-to-end of building height. The robot system can take place of this dangerous job. Second, the robotic system can save the labour cost. Staying in the air with only single rope is a great menace to human instinct. Therefore, only few people can get it as a job, and their labour cost is pretty high. Employers are hard up for hiring these workers. However, under the robotic system, only monitoring operators are needed. They can stay in a safe and comfort place.

The robot system of facade maintenance has two categories. First is a built-in type robot [1]. Rails are inserted to building facade to support the robot system. The robot navigates on the rail with good support, and well-designed utilities. However, the ready-inserted rails restrict the appearance of the building, and the robot system is often suitable only for the installed building.

\section{${ }^{1}$ Conference Extension}

- Sun, H., Kim, D. Y., Kwon, J. H., Kim, B.-S., and Park, C.-W. ,(2011) “The position and orientation measurement of gondola using a visual camera", in Proc. of the 2011 International Symposium on Automation and Robotics in Construction, Jul 2011, pp. 693-697.

- "Fusion with mechanical approach" is added. (25\% New Material)

DOI : $10.5121 /$ ijctcm.2012.2203 
Second is the gondola type robot [2]. The strong point of this type is that it can be installed at any rooftop. It enables a business model that cleaning service is provided to various buildings with some types of gondola robots. If well supported wire rope is bound, the gondola robot can operate mission on a high-rise building.

We concentrate on the gondola robot system. This type has following engineering problems. First, it is hard to recognize location of the robot because it holds only two wire ropes with heavy own weight. Second, the balance of the gondola cage is another requirement [3]. For painting tools, the direction of the spray trajectory is an important factor in order to procure its quality. Finally, the contact between the gondola cage and the building facade should be kept. The contact simplifies the kinematics of the gondola cage, but demands coping with obstacles on the walls, such as a security grille, terrace, and accessories of construction.

In this paper, a vision system is proposed as a methodology of sensing the pose of the gondola robot cage. We assume that the pose of the gondola cage is exposed with the wire rope attributes because the own weight of the gondola robot is so heavy enough to make the wire rope become a rigid body. We put a chess board on the rigid wire rope, and get six degree of freedom (6 DoF) of the chessboard. The $6 \mathrm{DoF}$ of chessboard coincide with the rope attribute and the pose of the gondola cage. At chapter 2, some preliminaries are introduced. Core description about our solution is suggested at chapter 3 . Chapter 4 proposes the fusion approach with mechanical approach. Some experiments is shown in chapter 5. Finally, chapter 6 concludes this paper.

\section{Preliminaries}

\subsection{Six degree of freedom $(6 \mathrm{DoF})$}

In three dimensional spaces, six values are used to describe translation and orientation of an object. First three numbers $x, y, z$ are the translation and location of the object in Cartesian coordinate. They define where the object is. Last three numbers $\alpha, \beta, \gamma$ are used for roll, pitch, and yaw, respectively. They notify the pose of the object. The poses are described as rotations around $x, y, z$ axis.

\subsection{Two dimensional chessboard}

Chessboard is a board with black and white grid colour, so it provides strong visual features. Therefore, it has been used for camera calibration [4,5]. With a pinhole or lens, the points on the chessboard in the three dimensional space undergo perspective transform. The parameters of the transform are expressed with a 3-by-3 homography matrix which contains rotation and translation information. The homography matrix is simply expressed as:

$$
\tilde{\mathbf{q}}=s \mathbf{H} \tilde{\mathbf{Q}}
$$

Here $\mathbf{q}$ and $\mathbf{Q}$ are a point in an image plane and an object plane in the real world respectively. $s$ is an scale factor. It is conventionally factored out of $\mathbf{H}$, which is homography matrix. 


\section{VISION-BASED 6 DOF EXTRACTING SYSTEM}

\subsection{System overview}

The proposed sensor system in this paper is designed to measure the pose of the gondola robot. Figure 1 is the concept of the sensor system. In order to hold the chessboard on the wire rope, a box is attached on the wire, and the wire rope pierces it. Because the gondola robot has two endless winders on its cage and the winders pulls the wire rope, the wire rope is just hanged on the gondola hanger. The pierced box is not moved as the wire rope is not moved when the gondola cage navigates. The chessboard on the pierced box is viewed by the vision camera on the gondola hanger (a rope cart).

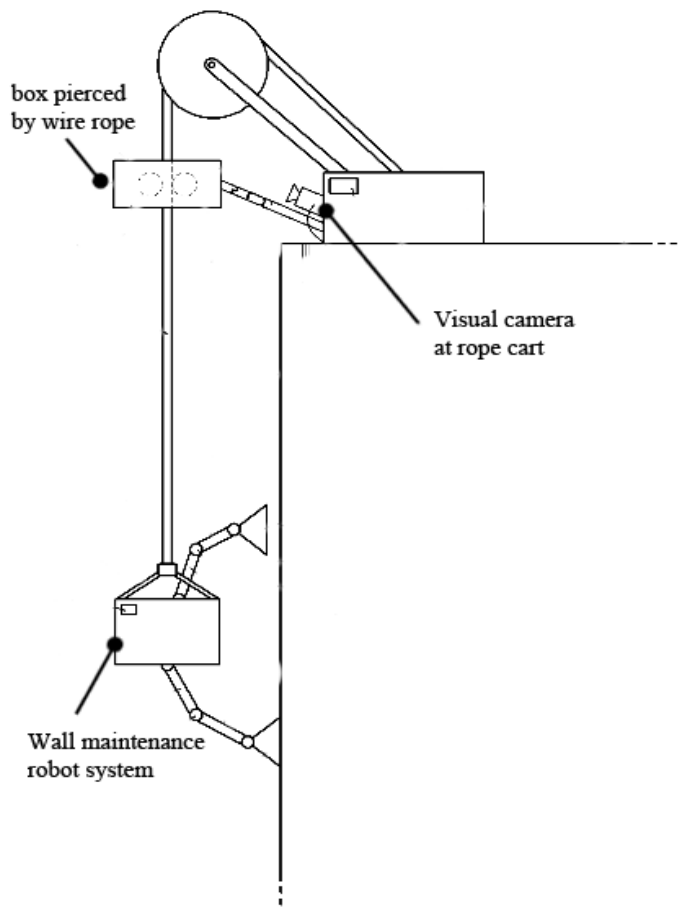

Figure 1. The concept of visual approach for sensing the pose of the gondola robot cage.

In accordance with the fluctuation of the gondola cage pose, the wire rope attributes alters. The tension on the wire rope makes to keep the same attributes from bottom to top of the wire rope. The attribute of top of the wire rope is sensed by the chessboard on the pierced box. Therefore, we can sense the pose of the gondola cage with 6 DoF of the chessboard.

Contrast to inertial measurement unit (IMU) sensors, the proposed sensor system has following strong points. First, IMU sensors (e.g. 3DM2 ) are more expensive than webcam. IMU is a sensor

${ }^{2}$ http://www.microstrain.com 
that makes fusion of accelerometer, gyro, and magnetometer. However, webcam is massproduced, and the cost shrinks a lot. Second, the IMU sensors depend on the magnetometer to indentify the yaw. It uses the earth magnetic field, and large metal parts distort the magnetic field. The gondola robot is also a heavy instrument, and it has a lot of metal parts. The proposed vision based sensor system is more suitable because it does not use the magnetic field.

\subsection{Getting rotation and translation matrix of chessboard}

As shown in [5], following is rotation and translation matrix of chessboard. At equation (1), $\mathbf{H}$ is equal to the camera intrinsic matrix $\mathbf{M}$ multiplied by a combination of the first two rotation matrix columns, $\mathbf{r}_{1}$ and $\mathbf{r}_{2}$, and the translation vector $\mathbf{t}$.

$$
\mathbf{H}=\left[\begin{array}{lll}
\mathbf{h}_{1} & \mathbf{h}_{2} & \mathbf{h}_{3}
\end{array}\right]=s \mathbf{M}\left[\begin{array}{lll}
\mathbf{r}_{1} & \mathbf{r}_{2} & \mathbf{t}
\end{array}\right]
$$

Here,

$$
\mathbf{M}=\left[\begin{array}{ccc}
f_{x} & 0 & c_{x} \\
0 & f_{y} & c_{y} \\
0 & 0 & 1
\end{array}\right]
$$

Also, it is expressed as:

$$
\begin{aligned}
& \mathbf{r}_{1}=\lambda \mathbf{M}^{-1} \mathbf{h}_{1} \\
& \mathbf{r}_{2}=\lambda \mathbf{M}^{-1} \mathbf{h}_{2} \\
& \mathbf{r}_{3}=\lambda \mathbf{M}^{-1} \mathbf{h}_{3}
\end{aligned}
$$

Here, $\lambda=\frac{1}{s}$.

Algebraically, a rotation matrix in $n$-dimensions is a $n$-by- $n$ special orthogonal matrix. Using the fact, we can get $\mathbf{r}_{3}$.

$$
\mathbf{r}_{3}=\mathbf{r}_{1} \times \mathbf{r}_{2}
$$

$\mathbf{M}$ and $\lambda$ are requires to get the rotation and translation matrix.

Becase $\mathbf{r}_{1}$ and $\mathbf{r}_{2}$ are orthonormal, the first constraint is:

$$
\begin{aligned}
& \mathbf{r}_{1}^{\mathrm{T}} \mathbf{r}_{\mathbf{2}}=\mathbf{0} \\
& \left(\lambda \mathbf{M}^{-1} \mathbf{h}_{1}\right)^{T}\left(\lambda \mathbf{M}^{-1} \mathbf{h}_{2}\right)=\mathbf{0} \\
& \mathbf{h}_{1}^{\mathrm{T}} \mathbf{M}^{-\mathrm{T}} \mathbf{M}^{-1} \mathbf{h}_{\mathbf{2}}=\mathbf{0}
\end{aligned}
$$

Here, $\mathbf{M}^{-\mathbf{T}}$ means $\left(\mathbf{M}^{-1}\right)^{\mathbf{T}}$.

The second constraint bases on the fact that the magnitudes of the rotation vectors are equal. 
International Journal of Control Theory and Computer Modelling (IJCTCM) Vol.2, No.2, March 2012

$$
\begin{aligned}
& \left\|\mathbf{r}_{1}\right\|=\left\|\mathbf{r}_{2}\right\| \\
& \mathbf{r}_{1}^{\mathrm{T}} \mathbf{r}_{1}=\mathbf{r}_{2}^{\mathrm{T}} \mathbf{r}_{2} \\
& \mathbf{h}_{1}^{\mathrm{T}} \mathbf{M}^{-\mathrm{T}} \mathbf{M}^{-1} \mathbf{h}_{1}=\mathbf{h}_{2}^{\mathrm{T}} \mathbf{M}^{-\mathrm{T}} \mathbf{M}^{-1} \mathbf{h}_{2}
\end{aligned}
$$

Let

$$
\begin{aligned}
\mathbf{B} & =\mathbf{M}^{-\mathbf{T}} \mathbf{M}^{-1}=\left[\begin{array}{lll}
B_{11} & B_{12} & B_{13} \\
B_{21} & B_{22} & B_{23} \\
B_{31} & B_{32} & B_{33}
\end{array}\right] \\
& =\left[\begin{array}{ccc}
\frac{1}{f_{x}^{2}} & 0 & -\frac{c_{x}}{f_{x}^{2}} \\
0 & \frac{1}{f_{y}^{2}} & -\frac{c_{y}}{f_{y}^{2}} \\
-\frac{c_{x}}{f_{x}^{2}} & -\frac{c_{y}}{f_{y}^{2}} & \frac{c_{x}^{2}}{f_{x}^{2}}+\frac{c_{y}^{2}}{f_{y}^{2}}+1
\end{array}\right]
\end{aligned}
$$

Two constraints have the general form.

$$
\begin{aligned}
& \mathbf{h}_{i}^{\mathbf{T}} \mathbf{B} \mathbf{h}_{\mathbf{j}} \\
&=\left(h_{i 1} B_{11}+h_{i 2} B_{21}+h_{i 3} B_{31}\right) h_{j 1} \\
&+\left(h_{i 1} B_{12}+h_{i 2} B_{22}+h_{i 3} B_{32}\right) h_{j 2} \\
&+\left(h_{i 1} B_{13}+h_{i 2} B_{23}+h_{i 3} B_{33}\right) h_{j 3} \\
&= h_{i 1} B_{11} h_{j 1}+h_{i 2} B_{21} h_{j 1}+h_{i 3} B_{31} h_{j 1} \\
&+h_{i 1} B_{12} h_{j 2}+h_{i 2} B_{22} h_{j 2}+h_{i 3} B_{32} h_{j 2} \\
&+h_{i 1} B_{13} h_{j 3}+h_{i 2} B_{23} h_{j 3}+h_{i 3} B_{33} h_{j 3}
\end{aligned}
$$

Becasue $\mathbf{B}$ is symmetric, $B_{12}=B_{21}, B_{13}=B_{31}, B_{23}=B_{32}$. Then, equation (9) is 
International Journal of Control Theory and Computer Modelling (IJCTCM) Vol.2, No.2, March 2012

$$
\begin{aligned}
= & h_{i 1} h_{j 1} B_{11}+\left(h_{i 1} h_{j 2}+h_{i 2} h_{j 1}\right) B_{12}+h_{i 2} h_{j 2} B_{22} \\
& +\left(h_{i 3} h_{j 1}+h_{i 1} h_{j 3}\right) B_{13} \\
& +\left(h_{i 3} h_{j 2}+h_{i 2} h_{j 3}\right) B_{23}+h_{i 3} h_{j 3} B_{33} \\
= & {\left[\begin{array}{c}
h_{i 1} h_{j 1} \\
h_{i 1} h_{j 2}+h_{i 2} h_{j 1} \\
h_{i 2} h_{j 2} \\
h_{i 3} h_{j 1}+h_{i 1} h_{j 3} \\
h_{i 3} h_{j 2}+h_{i 2} h_{j 3} \\
h_{i 3} h_{j 3}
\end{array}\right]\left[\begin{array}{c}
B_{11} \\
B_{12} \\
B_{22} \\
B_{13} \\
B_{23} \\
B_{33}
\end{array}\right]=\mathbf{v}_{i \mathbf{i j}}^{\mathbf{T}} }
\end{aligned}
$$

Using $\mathbf{v}_{\mathbf{i j}}^{\mathbf{T}}$, two constraints which are equation (6), equation (7) can be converted as

$$
\left[\begin{array}{c}
v_{12}^{T} \\
\left(v_{11}-v_{22}\right)^{T}
\end{array}\right] b=0
$$

If $n$ images of the chessboard are observed, by stacking $n$ such equations as equation (10) we have a follow equation.

$$
\mathbf{V b}=\mathbf{0}
$$

Here, $\mathbf{V}$ is a $2 n$-by-6 matrix. If $n \geq 3$, a unique solution $\mathbf{b}$ defined up to a scale factor is obtainable. The equation (11) is well known as the eigenvector of $\mathbf{V}^{\mathrm{T}} \mathbf{V}$ associated with the smallest eigen value.

\section{FUSION WITH MECHANICAL APPROACH}

We have researched the mechanical way to get the rope attribute with the RAM sensor [6]. Fig. 2 is a method applying the proposed vision approach in this paper to the RAM sensor. Fig. 2(a) is a camera that acquires images of the chessboard of RAM sensor. Fig. 2(b) is the chessboard which is held by the end-effecter of the RAM sensor and coincided with it. Fig. 2(c) are the absolute encoders that informs the angles of the passive joints. Fig. 2(d) is the start point of the RAM sensor kinematics. 


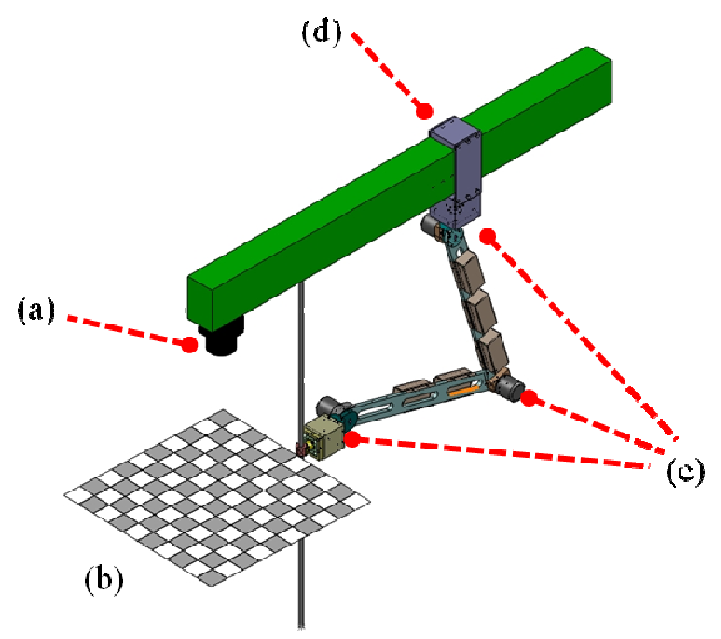

Figure 2. Usage of vision sensor at the RAM sensor.

The RAM sensor at Fig. 2 estimates the rope attribute in two ways. First, as in [5], the rope is sensed through the end effecter of the link with passive joints. The kinematics with the output of five absolute encoders calculates it. Second, the chessboard also shows the wire rope attributes. There are three ways to use above two ways. First is simple average of two values. Second, we can select one which is the most trustable. Finally, weighted sum can be applied in response to situation of the gondola.

\section{EXPERIMENT}

Fig. 3 is the experiment environment that has the pierced box with the chessboard. A webcam viewed the chessboard, and grabbed the chessboard images.

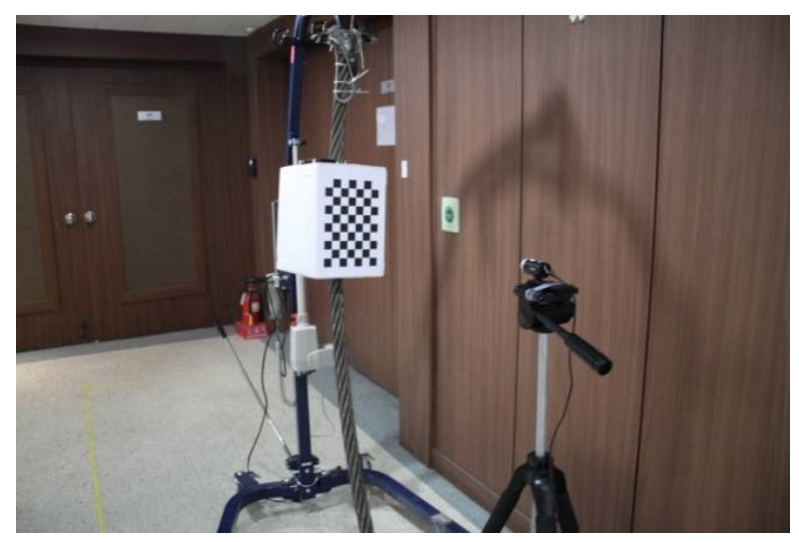

Figure 3. Experiment environment.

On this chessboard, there are 45 interior corners with the same spacing. Fig. 4 shows the result of corner detection. 
International Journal of Control Theory and Computer Modelling (IJCTCM) Vol.2, No.2, March 2012

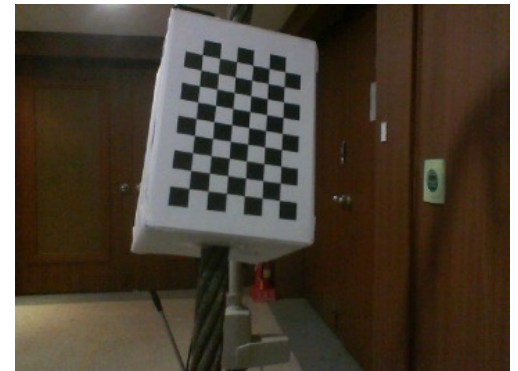

(a) Raw Image

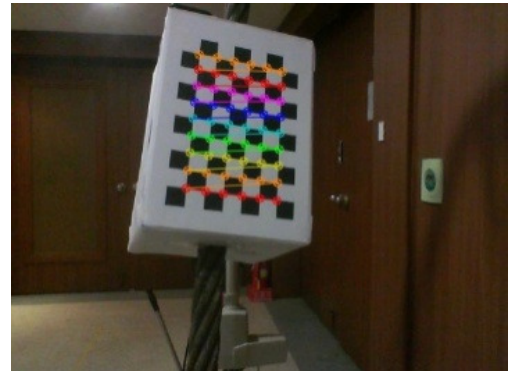

(b) Chessboard corners

Figure 4. Chessboard corner detection

Every rotation result of each scene is compared by gyro results. Fig. 5 shows the comparison.

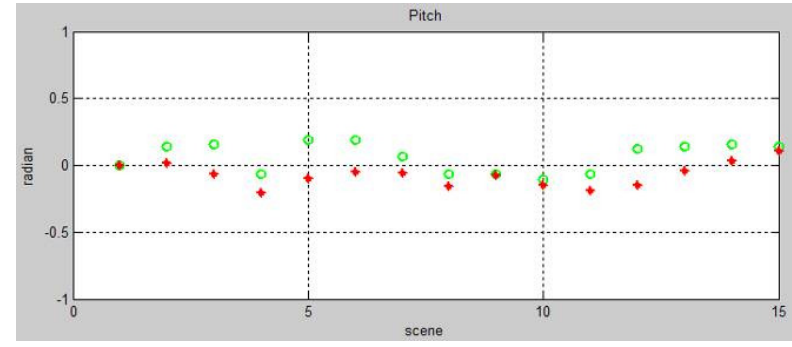

(a) Roll

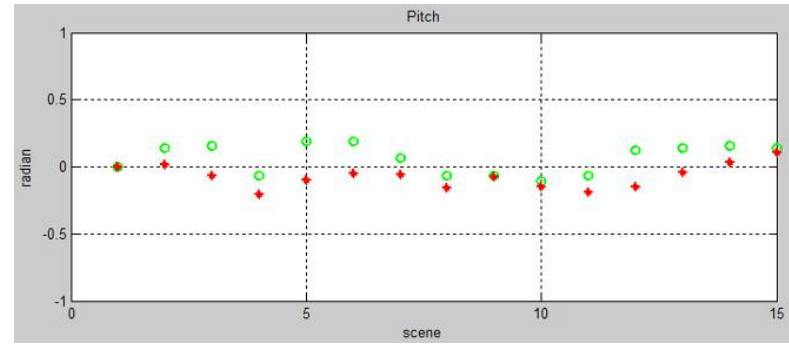

(b) Pitch

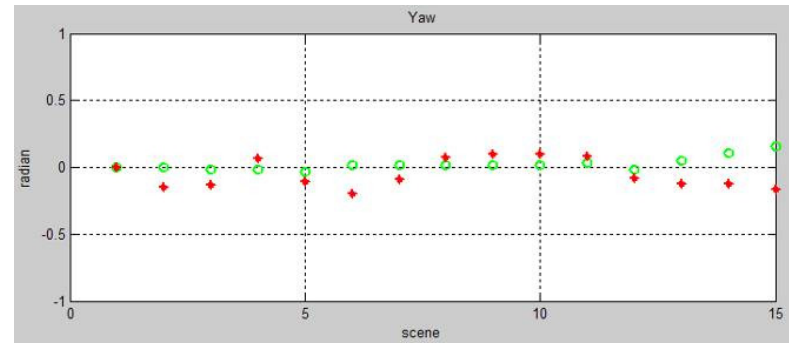

(c) Yaw

Figure 5. Error values

We got fifteen samples, and extracted their orientations. In order to compare the proposed sensor system and gyro sensor, the first sample is used as an origin. The values of samples are subtracted 
by the first sample vale, and the subtracted values are plotted on the figure 5 . For example, the fifth value of figure 5 is the gap of orientation between fifth sample value and first sample value. Also, figure 6 shows the error graph. The error means the difference between gyro sensor output and the proposed sensor value.

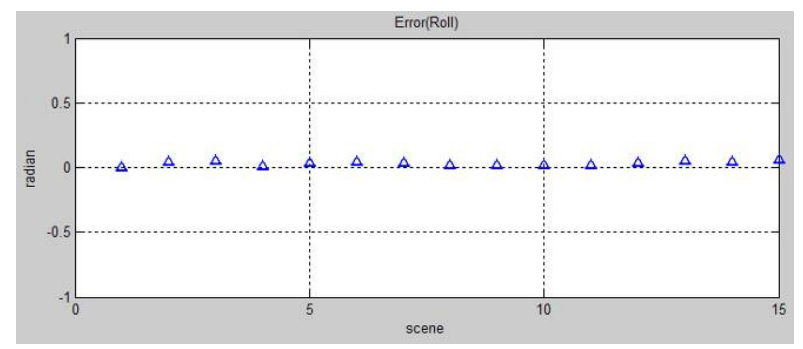

(a) Roll

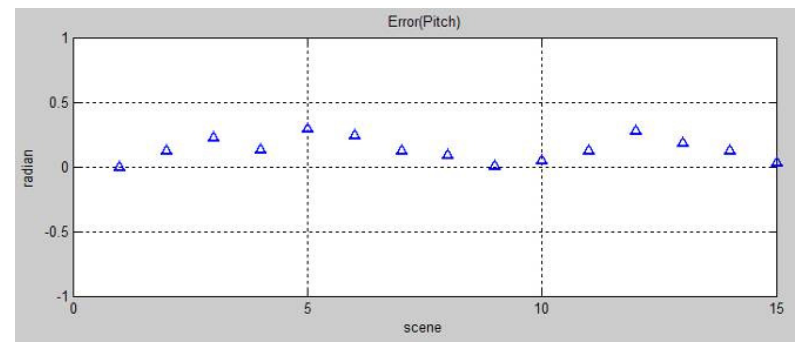

(b) Pitch

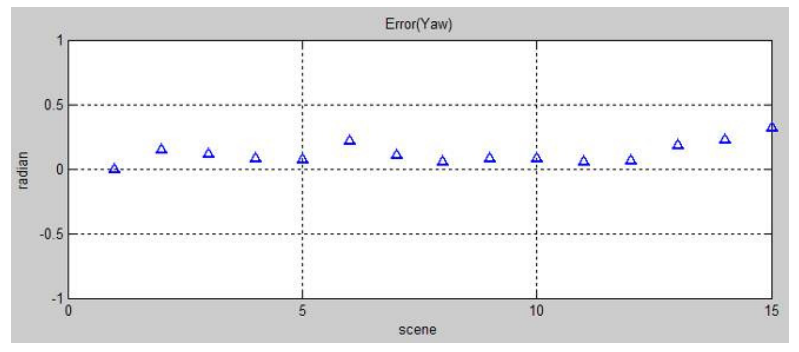

(c) Yaw

Figure 6. Error values

\section{CONCLUSiOnS}

The proposed sensor system in this paper is designed for automating the gondola type building facade maintenance robot. Its role is to sense the gondola cage pose because it is important when the gondola robot conducts its mission such as painting and monitoring the building facade.

Our sensor system is cheaper than an IMU sensor system, and robust to magnetic noise. Additionally, it can estimate translation information of the gondola cage if the distance from the gondola hanger and the gondola cage is provided. 
International Journal of Control Theory and Computer Modelling (IJCTCM) Vol.2, No.2, March 2012

In future, we will research other sensing systems for the gondola robot. Then, sensor fusion will be applied on them to make the sensing robust.

\section{ACKNOWLEDGEMENTS}

The work presented in this paper was funded by BMRC(Building-Façade Maintenance Robot Research Center), supported by Korea Institute of Construction and Transportation Technology Evaluation

\section{REFERENCES}

[1] Sack, M., Elkmann, N., Felsch, T., and Bohme, T., (2002) "Intelligent control of modular kinematics - the robot platform STRIUS", in Proc. of the 2002 IEEE International Symposium on Intelligent Control, 2002, pp. 549-553.

[2] Zhang, H., Zhang, J., and Zong, G., (2004) "Requirements of glass cleaning and development of climbing robot systems", in Proc. of the 2004 International Conference on Intelligent Mechatronics and Automation, Aug 2004, pp. 101-106.

[3] Vaidyanathan, S., (2011) "Global synchronization of four-wing chaotic systems by sliding mode control”, International Journal of Control Theory and Computer Modelling (IJCTCM), Vol. 1, No. 1, Jul 2011.

[4] Zhang, R., Tsi, P.-S., Cryer, J. E., and Shah, M. (1999) "Shape form shading: A survey", IEEE Transactions on Pattern Analysis and Machine Intelligence 21 (1999): 690-706

[5] Zhang, Z., (2000) “A flexible new technique for camera calibration”, IEEE Transactions on Pattern Analysis and Machine Intelligence 22 (2000): 1330-1334

[6] Kim, D. Y., Kwon, J. H., Sun, H., Kim, B.-S., and Park, C.-W. , (2011) "Rope attribute measurement system for gondola type facade robot", in Proc. of the 2011 2nd International Conference on Construction and Project Management (ICCPM 2011), Sep 2011, pp. 103-106. 
International Journal of Control Theory and Computer Modelling (IJCTCM) Vol.2, No.2, March 2012

\section{Authors}

Hwadong Sun received the B.S. degree in computer science from Paichai University, Daejeon, Korea, in 2005, and the M.S. degree in electrical and electronic engineering from Yonsei University, Seoul, in 2011. He has been a researcher in Korea Electronics Technology Institute, Gyeonggi-do, Korea, under the Ministry of Knowledge Economy since 2003. His current research interests include SLAM, field robotics, and robot vision.

Joon ho Kwon received the B.S. degree in Department of Information and Control Engineering from Kwangwoon University, Seoul, Korea, in 2011. He is a M.S. candidate in Mechanical Engineering at Korea University. He has been a researcher in Korea Electronics Technology Institute under the Ministry of Knowledge Economy since 2008. His current research interests include embedded control and motor control.

Kim, Dong Yeop received the B.S. and M.S. degree in School of Electrical \& Electronic Engineering at Yonsei University, Seoul, Korea, in 2008 and 2010, respectively.He has been a researcher in Korea Electronics Technology Institute under the Ministry of Knowledge Economy since 2010. His current research interests include intelligent systems, SLAM, field robotics, and robot vision.

Bong-Seok Kim received the B.S degree in Aeronautical Mechanical Engineering at Korea Aerospace University, Seoul, Korea, and M.S degree in Mechanical Engineering at Korea University. He is a Ph.D. candidate in Mechanical Engineering at Korea University. He has been a senior researcher in Korea Electronics Technology Institute under the Ministry of Knowledge Economy since 2005. His current research interests include mechanical design and control of robot manipulator.

Chang-Woo Park received the B.S. degree in Electronics Eng. from Korea University in 1997 and M.S. and Ph.D. degrees in Electronics Eng. from Yonsei University, Seoul, Korea, in 1999 and 2003, respectively. He has been a managerial researcher in Korea Electronics Technology Institute under the Ministry of Knowledge Economy since 2003. His current research interests include intelligent systems, nonlinear control, fuzzy systems, robot vision and robotics. 